PROCEEDINGS OF THE

AMERICAN MATHEMATICAL SOCIETY

Volume 135, Number 11, November 2007, Pages 3553-3560

S 0002-9939(07)08913-7

Article electronically published on June 21, 2007

\title{
ON THE LOCAL HÖLDER CONTINUITY OF THE INVERSE OF THE $p$-LAPLACE OPERATOR
}

\author{
AN LE
}

(Communicated by David S. Tartakoff)

\begin{abstract}
We prove an interpolation type inequality between $C^{\alpha}, L^{\infty}$ and $L^{p}$ spaces and use it to establish the local Hölder continuity of the inverse of the $p$-Laplace operator: $\left\|\left(-\Delta_{p}\right)^{-1}(f)-\left(-\Delta_{p}\right)^{-1}(g)\right\|_{C^{1}(\bar{\Omega})} \leq C\|f-g\|_{L^{\infty}(\Omega)}^{r}$, for any $f$ and $g$ in a bounded set in $L^{\infty}(\Omega)$.
\end{abstract}

\section{INTRODUCTION}

Let $\Omega$ be a bounded domain in $\mathbb{R}^{N}$ with $C^{1, \gamma}$ boundary and $1<p<\infty$. Consider the Dirichlet problem

$$
\begin{cases}-\Delta_{p} u & =f, \text { in } \Omega, \\ u & =0, \text { on } \partial \Omega,\end{cases}
$$

where $-\Delta_{p} u=-\operatorname{div}\left(|\nabla u|^{p-2} \nabla u\right)$ is the $p$-Laplace operator. The solution $u$ to (1.1) is unique for a given $f$ in $L^{\infty}(\Omega)$. It follows from regularity results in [2] and [3] that the inverse of the $p$-Laplace operator

$$
L:=\left(-\Delta_{p}\right)^{-1}: L^{\infty}(\Omega) \rightarrow C^{1, \alpha}(\bar{\Omega})
$$

is a continuous and compact mapping for some $0<\alpha<1$. We write $u=L f$ if $u$ solves (1.1) in the weak sense (see (2.1) for the definition of weak solutions).

In this paper we study the Hölder continuity of the operator $L$. As we will see in section 2, one obtains $\|L f-L g\|_{\infty} \leq C\|f-g\|_{\infty}^{\frac{1}{p-1}}$, for $p$ large, by using Sobolev embedding theorems.

However, if we want to estimate the $C^{1}(\bar{\Omega})$ norm of $(L f-L g)$ in terms of $\|f-g\|_{\infty}$, then Sobolev embeddings do not give the result. It is so because $W^{1, p}(\Omega)$ is not a subset of $C^{1}(\bar{\Omega})$.

Our idea is to establish an interpolation inequality between $C^{1, \alpha}(\bar{\Omega}), C^{1}(\bar{\Omega})$ and $W^{1, p}(\Omega)$. Such an inequality together with the compactness of the operator $L$ gives us an estimate for $\|L f-L g\|_{C^{1}}$ and thus shows that $L$ is locally Hölder continuous.

Received by the editors December 1, 2005 and, in revised form, August 4, 2006.

2000 Mathematics Subject Classification. Primary 35J60, 35B65; Secondary 46B70.

Key words and phrases. p-Laplace operator, interpolation inequalities, Hölder continuity.

(C)2007 American Mathematical Society Reverts to public domain 28 years from publication 
We use the following notation:

$$
\begin{aligned}
& \alpha, \beta, \gamma: \text { positive constants in }(0,1) . \\
& U: \text { an open set in } \mathbb{R}^{N} \text {. } \\
& \Omega \quad \text { : an open bounded domain in } \mathbb{R}^{N} \text { with } C^{1, \gamma} \text { boundary. } \\
& c, r, \theta: \text { positive constants depending only on the universal } \\
& \text { parameters such as } N, p, \Omega \text {. } \\
& c(M) \quad \text { : a constant depending on } M \text { and possibly on } \\
& \text { the universal parameters. } \\
& C^{\alpha}(\bar{U}), C^{\alpha}\left(\mathbb{R}^{N}\right) \quad: \quad\left\{u:|u|_{\alpha}:=\sup _{x \neq y} \frac{|u(x)-u(y)|}{|x-y|^{\alpha}}<\infty\right\} . \\
& \|u\|_{C^{0, \alpha}}=\|u\|_{0, \alpha}=\|u\|_{\infty}+|u|_{\alpha} . \\
& C^{1}(\bar{U}), C^{1}\left(\mathbb{R}^{N}\right) \quad: \quad\{u: \nabla u \text { is continuous }\} . \\
& \|u\|_{C^{1}}=\|u\|_{1,0}=\|\nabla u\|_{\infty}+\|u\|_{\infty} .
\end{aligned}
$$

We denote by $\|\cdot\|_{1, \alpha}$ and $\|\cdot\|_{q}$ the norms in $C^{1, \alpha}$ and $L^{q}, 1 \leq q \leq \infty$, respectively. The spaces $W_{0}^{1, p}$ and $W^{1, p}, 1<p<\infty$, are used in the standard way.

Our first result is about an interpolation inequality in $\mathbb{R}^{N}$.

Theorem 1.1. Given $0<\alpha<1$ and $1 \leq q<\infty$, there exist constants $c$ and $0<\theta<1$ (depending only on $N, p$ and $\alpha$ ) such that for any $u$ in $C^{\alpha}\left(\mathbb{R}^{N}\right) \cap L^{q}\left(\mathbb{R}^{N}\right)$,

$$
\|u\|_{\infty} \leq c\|u\|_{0, \alpha}^{1-\theta}\|u\|_{q}^{\theta}
$$

Next, we establish the inequality (1.3) for an open set $U$ in $\mathbb{R}^{N}$. It is possible if $U$ satisfies the cone property (see Definition 3.1).

Theorem 1.2. Let $U$ be an open set in $\mathbb{R}^{N}$ such that $U$ satisfies the cone property. Given $0<\alpha<1$ and $1 \leq q<\infty$, there exist constants $c$ and $0<\theta<1$ such that for any $u$ in $C^{\alpha}(\bar{U}) \cap L^{q}(U)$

$$
\|u\|_{\infty} \leq c\|u\|_{0, \alpha}^{1-\theta}\|u\|_{q}^{\theta}
$$

An immediate consequence of Theorem 1.2 is the interplay between $C^{1, \alpha}(\bar{U})$, $C^{1}(\bar{U})$ and $W^{1, p}(U)$.

Corollary 1.3. Let $U$ be an open set in $\mathbb{R}^{N}$ such that $U$ satisfies the cone property. There exist constants $c>0$ and $0<\theta<1$ such that for any $u \in C^{1, \alpha}(\bar{U}) \cap W^{1, p}(U)$,

$$
\|u\|_{1,0} \leq c\|u\|_{1, \alpha}^{1-\theta}\|u\|_{W^{1, p}}^{\theta} .
$$

Inequality (1.5) is the key result which is used to show the Hölder continuity of the operator $L$ defined by (1.2). Let $B_{M}^{\infty}$ be a ball in $L^{\infty}(\Omega)$ with radius $M>0$. By compactness, the image $L\left(B_{M}^{\infty}\right)$ is a bounded set in $C^{1, \alpha}(\bar{\Omega})$.

Theorem 1.4. Given $M>0$, then there exist positive constants $c(M)$ and $r$ such that

$$
\|L f-L g\|_{1,0} \leq c(M)\|f-g\|_{\infty}^{r},
$$

for any $f$ and $g$ in $B_{M}^{\infty}$ (i.e. $0<\|f\|_{\infty},\|g\|_{\infty}<M$ ). 
Theorem 1.4 shows that the operator $L$, as a mapping from $L^{\infty}(\Omega)$ to $C^{1}(\bar{\Omega})$, is locally Hölder continuous if $0<r<1$ and is locally Lipschitz continuous if $r \geq 1$. The rest of the paper is organized as follows. In section 2 we recall preliminary results and study the $\|L f-L g\|_{\infty}$ estimate of problem (1.1). In section 3, we give the proofs of Theorem 1.1 and Theorem 1.2. Finally in section 4, we establish the Hölder continuity of the operator $L$ by proving Theorem 1.4 .

\section{Preliminary Results}

Let us recall the definition of weak solutions to problem (1.1). Given a measurable function $f$, we say a function $u$ in $W_{0}^{1, p}(\Omega)$ solves (1.1) in the weak sense provided that

$$
\int_{\Omega}|\nabla u|^{p-2} \nabla u \nabla v d x=\int_{\Omega} f v d x, \forall v \in W_{0}^{1, p}(\Omega) .
$$

Evidently, if $f$ is in $L^{q}(\Omega)$, for any $q \in[p, \infty]$, then the left hand side of (2.1) is finite for any $v$ in $W_{0}^{1, p}(\Omega)$.

In fact if $f$ is a bounded function, the corresponding solution $u$ is unique and differentiable. The following proposition is a summary of regularity theory of the p-Laplace operator.

Proposition 2.1. Let $f$ be in $L^{q}(\Omega)$ for some $q \in[p, \infty]$. Then the exists a unique solution $u$ to problem (1.1), i.e. the inverse p-Laplace operator $L$ is well defined. Moreover,

(i) If $q>\frac{N p}{p-1}$, then $u$ is in $C^{1, \beta}(\Omega)$ locally, i.e. $u$ is of class $C^{1, \beta}$ on any compact subset of $\Omega$.

(ii) If $q=\infty$, then $u$ is in $C^{1, \alpha}(\bar{\Omega})$. Furthermore, for any number $M$ positive there exists a constant $c=c(M)$ such that

$$
\|u\|_{1, \alpha}=\|L f\|_{1, \alpha} \leq c(M)
$$

whenever $0 \leq\|f\|_{\infty} \leq M$.

(iii) The operator $\bar{L}$ is continuous and compact from $L^{\infty}$ to $C^{1}(\bar{\Omega})$.

We recall that a mapping is said to be compact if it maps bounded sets to precompact sets.

Proof. The existence of a solution $u$ can be established by minimizing in $W_{0}^{1, p}(\Omega)$ the functional

$$
J(u)=\frac{1}{p} \int_{\Omega}|\nabla u|^{p} d x-\int_{\Omega} f u d x .
$$

The uniqueness of $u$ is a direct consequence of Lemma 2.2. Assertion (i) is a special case of the results proved in [2] or in [6] and assertion (ii) is shown in [3]. Finally, due to (ii) the operator $L$ maps bounded sets in $L^{\infty}(\Omega)$ to bounded sets in $C^{1, \alpha}(\bar{\Omega})$. Consequence, we obtain (iii) by the compact embedding from $C^{1, \alpha}(\bar{\Omega})$ into $C^{1}(\bar{\Omega})$.

In fact, $L$ is a continuous and compact mapping from $L^{\infty}(\Omega)$ to $C^{1, \alpha^{\prime}}(\bar{\Omega})$, for any $0 \leq \alpha^{\prime}<\alpha$. 
Let $A$ be the differential mapping of the functional $\frac{1}{p} \int_{\Omega}|\nabla u|^{p} d x$,

$$
\begin{aligned}
A & : \quad W_{0}^{1, p}(\Omega) \rightarrow W_{0}^{1, p}(\Omega)^{*}, \\
\langle A u, v\rangle & =\int_{\Omega}|\nabla u|^{p-2} \nabla u \nabla v d x, \forall u, v \in W_{0}^{1, p}(\Omega) .
\end{aligned}
$$

Clearly, $u=L f$ is equivalent to $\langle A u, \cdot\rangle=\langle f, \cdot\rangle_{L^{2}}$.

In the proof of Theorem 1.4 we use some properties of the operator $A$. In [5], Lindqvist shows that there exists a constant $c=c(p)$ such that for any vector $x$ and $y$ in $\mathbb{R}^{N}$, one has

$$
\begin{aligned}
& |y|^{p} \geq|x|^{p}+p|x|^{p-2} x \cdot(y-x)+c|x-y|^{p}, \text { if } p \geq 2, \\
& |y|^{p} \geq|x|^{p}+p|x|^{p-2} x \cdot(y-x)+c \frac{|x-y|^{2}}{(|x|+|y|)^{2-p}}, \text { if } 1<p<2 .
\end{aligned}
$$

Direct calculations yield the following:

Lemma 2.2. For any $u$ and $v$ in $W_{0}^{1, p}(\Omega)$, we have

$$
\begin{aligned}
& \langle A u-A v, u-v\rangle \geq c(p) \int_{\Omega}|\nabla u-\nabla v|^{p} d x, \text { if } p \geq 2, \\
& \langle A u-A v, u-v\rangle \geq c(p) \int_{\Omega} \frac{|\nabla u-\nabla v|^{2}}{(|\nabla u|+|\nabla v|)^{2-p}} d x, \text { if } 1<p<2 .
\end{aligned}
$$

Equalities hold if and only if $u=v$ in $\Omega$.

To motivate our work, let us estimate $\|L f-L g\|_{\infty}$. Using (2.3) in Lemma 2.2 one can show that for $p>N$,

$$
\|L f-L g\|_{\infty} \leq c\|f-g\|_{\infty}^{\frac{1}{p-1}} .
$$

To see this, let $u=L f$ and $v=L g$. It follows from (2.3) that

$$
c\|u-v\|_{W_{0}^{1, p}(\Omega)}^{p} \leq\langle A u-A v, u-v\rangle=\int_{\Omega}(f-g)(u-v),
$$

which, by Hölder inequality, implies

$$
c\|u-v\|_{W_{0}^{1, p}(\Omega)}^{p} \leq\|f-g\|_{\infty}\|u-v\|_{W_{0}^{1, p}(\Omega)} .
$$

As $p>N$, the space $W_{0}^{1, p}(\Omega)$ is compactly embedded into $L^{\infty}(\Omega)$. Thus, (2.5) follows.

Remark 2.3. (i) As we see the from the argument, the constant $c$ in (2.5) does not depend on $f$ and $g$. (ii) Using the identity $L(k f)=k^{\frac{1}{p-1}} L f$, for any $k>0$, we obtain from Proposition 2.1(ii) that there exists a constant $c$ independent of $f$ such that

$$
\|L f\|_{1, \alpha} \leq c\|f\|_{\infty}^{\frac{1}{p-1}}
$$

\section{INTERPOLATION INEQUALITIES}

In this section we establish interpolation inequalities between $C^{\alpha}, L^{\infty}$ and $L^{q}$ in $\mathbb{R}^{N}$ and in its open subsets. 
Proof of Theorem 1.1. If $\|u\|_{\infty}=\infty$, then $\|u\|_{0, \alpha}=\infty$ and (1.3) holds. Suppose $\|u\|_{\infty}<\infty$, it suffices to show

$$
|u(0)| \leq c\|u\|_{0, \alpha}^{1-\theta}\|u\|_{q}^{\theta}
$$

Since for arbitrary $y$ in $\mathbb{R}^{N}$, we let $v(x)=u(x+y)$ and use (3.1) to obtain

$$
|u(y)|=|v(0)| \leq c\|u\|_{0, \alpha}^{1-\theta}\|u\|_{q}^{\theta},
$$

which implies (1.3).

To see (3.1) we first observe from the triangle inequality that

$$
\begin{aligned}
|u(0)| & \leq \frac{|u(x)-u(0)|}{|x|^{\alpha}}|x|^{\alpha}+|u(x)| \\
& \leq\|u\|_{0, \alpha}|x|^{\alpha}+|u(x)|, \quad \forall x \in \mathbb{R}^{N} .
\end{aligned}
$$

For any $r>0$, we integrate both sides of (3.2) over the ball $B_{r}=B(0, r)$ to get

$$
\begin{aligned}
\omega r^{N}|u(0)| & \leq\|u\|_{0, \alpha} \int_{B_{r}}|x|^{\alpha} d x+\int_{B_{r}}|u(x)| d x \\
& \leq \frac{N}{N+\alpha} \omega r^{N+\alpha}\|u\|_{0, \alpha}+\left(\omega r^{N}\right)^{1 / q^{\prime}}\|u\|_{L^{q}\left(B_{r}\right)} .
\end{aligned}
$$

Here $\omega$ is the volume of the unit ball in $\mathbb{R}^{N}$ and $\frac{1}{q^{\prime}}=1-\frac{1}{q}$ if $q>1, \frac{1}{q^{\prime}}=0$ if $q=1$. Thus,

$$
\begin{aligned}
|u(0)| & \leq \frac{N}{N+\alpha} r^{\alpha}\|u\|_{0, \alpha}+\left(\omega r^{N}\right)^{-1+1 / q^{\prime}}\|u\|_{q} \\
& \leq c\left(r^{\alpha}\|u\|_{0, \alpha}+\frac{1}{r^{\beta}}\|u\|_{q}\right),
\end{aligned}
$$

where $c=\max \left\{\frac{N}{N+\alpha}, \omega^{-1+1 / q^{\prime}}\right\}$ and $\beta=N\left(1-1 / q^{\prime}\right)>0$. We note that the above inequality holds for any $r>0$. We use the fact

$$
r^{\alpha} a+\frac{1}{r^{\beta}} b=a^{1-\theta} b^{\theta}, \text { if } r^{\alpha+\beta}=\frac{b}{a} \text { and } \theta=\frac{\alpha}{\alpha+\beta},
$$

to conclude that

$$
|u(0)| \leq c\|u\|_{0, \alpha}^{1-\theta}\|u\|_{q}^{\theta},
$$

with $\theta=\frac{\alpha}{\alpha+\beta}$.

The main idea in the previous proof is the integration over any ball $B_{r}$, with arbitrary radius $r>0$, both sides of inequality (3.2). Given $U$ an open set in $\mathbb{R}^{N}$, we want to establish (1.4) (in Theorem 1.2) by using such an idea. By translation, we can assume 0 is in $\bar{U}$, and thus we obtain (3.2) for any $x \in U$. However, $U$ may not contain all the balls $B_{r}=B(0, r)$ with arbitrary radius, or $U$ may not even contain any ball $B_{r}$ at all if 0 is on the boundary of $U$. As we will see later, it turns out that we only need to integrate on a fixed portion of a ball, namely a cone, and up to a fixed radius $r_{0}$. For this reason, we assume that $U$ satisfies the cone property.

Definition 3.1. We say an open $U$ satisfies the cone property if there is a cone $K\left(r_{0}\right)$ with radius $r_{0}>0$ such that for each point $x$ in $\bar{U}$ we can place inside $\bar{U}$ a cone $K\left(x, r_{0}\right)$, center at $x$, which is congruent to the cone $K\left(r_{0}\right)$. 
Proof of Theorem 1.2. It suffices to show, for any $x$ in $\bar{U}$, that

$$
|u(x)| \leq c\|u\|_{0, \alpha}^{1-\theta}\|u\|_{q}^{\theta} .
$$

Following the argument made in the proof of Theorem 1.1 we obtain

$$
|u(x)| \leq\|u\|_{0, \alpha}|y-x|^{\alpha}+|u(y)|,
$$

for any $y$ in $\bar{U}$. Let $K\left(x, r_{0}\right)$ be the cone center at $x$ (by Definition 3.11). For any $0<r<r_{0}$, integrating both sides of (3.4) over the cone $K(r)=K\left(x, r_{0}\right) \cap B(x, r)$ to get

$$
\begin{aligned}
\omega_{K} r^{N}|u(x)| & \leq\|u\|_{0, \alpha} \int_{K(r)}|y-x|^{\alpha} d y+\int_{K(r)}|u(y)| d y \\
& \leq \frac{N}{N+\alpha} \omega_{K} r^{N+\alpha}\|u\|_{0, \alpha}+\left(\omega_{K} r^{N}\right)^{1 / q^{\prime}}\|u\|_{L^{q}\left(B_{r}\right)},
\end{aligned}
$$

here $\omega_{K}=|K(0,1)|$, the volume of $\frac{1}{r_{0}^{N}} K\left(r_{0}\right)$. Thus there is a constant $c_{1}>0$ such that

$$
|u(x)| \leq c_{1}\left(r^{\alpha}\|u\|_{0, \alpha}+\frac{1}{r^{\beta}}\|u\|_{q}\right),
$$

for any $0<r<r_{0}$. Let $\theta=\frac{\alpha}{\alpha+\beta}$; we consider two cases.

(i) $\frac{\|u\|_{q}}{\|u\|_{0, \alpha}}<r_{0}^{\alpha+\beta}$. By taking $r^{\alpha+\beta}=\frac{\|u\|_{q}}{\|u\|_{0, \alpha}}$ and substituting into the above inequality we obtain

$$
|u(x)| \leq c_{1}\|u\|_{0, \alpha}^{1-\theta}\|u\|_{p}^{\theta} .
$$

(ii) $\frac{\|u\|_{q}}{\|u\|_{0, \alpha}} \geq r_{0}^{\alpha+\beta}$. We note that

$$
\|u\|_{\infty} \leq\|u\|_{0, \alpha} \leq\|u\|_{0, \alpha}^{1-\theta}\|u\|_{0, \alpha}^{\theta} \leq\|u\|_{0, \alpha}^{1-\theta}\|u\|_{q}^{\theta} r_{0}^{\alpha} .
$$

Combining these two cases we conclude (3.3) with $c=\max \left\{c_{1}, r_{0}^{\alpha}\right\}$.

We leave the verification of Corollary 1.3 to the readers.

\section{On the local Hölder CONTINUity of $L$}

As mentioned in section 1 , we assume $\Omega$ is a bounded domain in $\mathbb{R}^{N}$ with $C^{1, \gamma}$ boundary and $1<p<\infty$. Evidently, $\Omega$ satisfies the cone property. Let us recall that $u=L f$ if and only if $\langle A u, \cdot\rangle=\langle f, \cdot\rangle_{L^{2}}$ where the operators $L$ and $A$ are defined by (1.2) and by (2.2), respectively.

Proof of Theorem 1.4. Let $f$ and $g$ be in $B_{M}^{\infty}$, the ball with radius $M$ in $L^{\infty}(\Omega)$. We will show that there exist positive constants $r>0$ and $c=c(M)$ such that

$$
\|u-v\|_{1,0} \leq c\|f-g\|_{\infty}^{r}
$$

where $u=L f$ and $v=L g$.

It is clear that

$$
\langle A u-A v, u-v\rangle=\int_{\Omega}(f-g)(u-v) d x .
$$

We first establish (4.1) for the case $p \geq 2$. By inequality (2.3) in Lemma 2.2.

$$
c \int_{\Omega}|\nabla u-\nabla v|^{p} d x \leq\langle A u-A v, u-v\rangle .
$$


Using Hölder's inequality, we can estimate from above the right hand side of (4.2)

$$
\int_{\Omega}(f-g)(u-v) d x \leq c\|f-g\|_{\infty}\|u-v\|_{W_{0}^{1, p}}
$$

Thus,

$$
\|u-v\|_{W_{0}^{1, p}}^{p-1} \leq c\|f-g\|_{\infty} .
$$

On the other hand, as $u-v$ is in $C^{1, \alpha}(\bar{\Omega})$, it follows from (1.5) in Corollary 1.3 that

$$
\|u-v\|_{1,0} \leq c\|u-v\|_{1, \alpha}^{1-\theta}\|u-v\|_{W_{0}^{1, p}(\Omega)}^{\theta} .
$$

By the boundedness of $L$ (Proposition 2.1(ii)),

$$
\|u-v\|_{1, \alpha} \leq\|u\|_{1, \alpha}+\|v\|_{1, \alpha} \leq c(M) .
$$

Combining (4.3), (4.4) and (4.5) we conclude that

$$
\|u-v\|_{1,0} \leq c(M)\|u-v\|_{W_{0}^{1, p}(\Omega)}^{\theta} \leq c(M)\|f-g\|_{\infty}^{\frac{\theta}{p-1}},
$$

which is (4.1) with $r=\frac{\theta}{p-1}$.

If $1<p<2$, we use inequality (2.4),

$$
c(p) \int_{\Omega} \frac{|\nabla u-\nabla v|^{2}}{(|\nabla u|+|\nabla v|)^{2-p}} d x \leq\langle A u-A v, u-v\rangle,
$$

(4.2) and the boundedness of $L$ to obtain

$$
\int_{\Omega}|\nabla u-\nabla v|^{2} d x \leq c(M) \int_{\Omega}(f-g)(u-v) d x .
$$

By Hölder inequality, the analog to (4.3) in this case is

$$
\|u-v\|_{W_{0}^{1,2}} \leq c(M)\|f-g\|_{\infty} .
$$

We carry the same argument made in the previous case with $p=2$ to conclude (4.1).

We close the paper with some remarks.

Remark 4.1. The fact that the solution operator $L$ is continuous (Proposition 2.1(iii)) is not new. In fact it is a direct consequence of Proposition 2.1(ii), which is proved in 3]. Yet the Hölder continuity of $L$ says more about the stability of solutions with respect to data. Inequality (1.6) gives an estimate on the rate of convergence of solutions in $C^{1}$ spaces.

Remark 4.2. It is not clear to us that $L$ is actually (globally) Hölder continuous from $L^{\infty}(\Omega)$ to $C^{1}(\bar{\Omega})$. In other words, it may be possible that the constant $c$ in (1.6) and in (4.1) does not depend on $M$. This is the case if $p=2$ or if $N=1$ or if we consider radial solutions of problem (1.1) in the unit ball of $\mathbb{R}^{N}$. Also, as we note in Remark 2.3. inequalities (2.5) and (2.6) hold globally.

Remark 4.3. While reading the manuscript, the referee has noticed that condition $q>\frac{N p}{p-1}$ in Proposition 2.1(i) can be replaced by $q>N$. The idea is to use the arguments in Section 5 of $[4$ with the measure $\mu$ having Radon-Nikodym derivative equal to $f$. Also, as pointed out by the referee, Lemma 3.1 in [1] is very close to Theorem 1.1 . 


\section{ACKNOWLEDGMENTS}

The author is grateful to Professor Hai Dang for proposing a problem which led to the current work. The author thanks the referee for carefully reading the manuscript, for many helpful comments and especially for his insightful remark improving the condition in Proposition 2.1. The author also thanks Professor Lawrence C. Evans, Professor Neil Trudinger, Professor Klaus Schmitt and Professor Lihe Wang for many interesting discussions. This work was done while the author was visiting MSRI in Berkeley, California, in the Fall of 2005.

\section{REFERENCES}

[1] Luis A. Caffarelli and Qingbo Huang, Estimates in the generalized Campanato-JohnNirenberg spaces for fully nonlinear elliptic equations, Duke Math. J. 118 (2003), no. 1, 1-17. MR.1978880 (2004b:35082)

[2] E. DiBenedetto, $C^{1+\alpha}$ local regularity of weak solutions of degenerate elliptic equations, Nonlinear Anal. 7 (1983), no. 8, 827-850. MR709038 (85d:35037)

[3] Gary M. Lieberman, Boundary regularity for solutions of degenerate elliptic equations, Nonlinear Anal. 12 (1988), no. 11, 1203-1219. MR969499 (90a:35098)

[4] Sharp forms of estimates for subsolutions and supersolutions of quasilinear elliptic equations involving measures, Comm. Partial Differential Equations 18 (1993), no. 7-8, 11911212. MR 1233190 (94g:35088)

[5] Peter Lindqvist, Addendum: "On the equation $\operatorname{div}\left(|\nabla u|^{p-2} \nabla u\right)+\lambda|u|^{p-2} u=0$ " [Proc. Amer. Math. Soc. 109 (1990), 157-164], Proc. Amer. Math. Soc. 116 (1992), no. 2, 583584. MR:1007505 (90h:35088)

[6] Peter Tolksdorf, Regularity for a more general class of quasilinear elliptic equations, J. Differential Equations 51 (1984), no. 1, 126-150. MR727034 (85g:35047)

Mathematics Sciences Research Institute, 17 Gauss Way, Berkeley, California 794720 Current address: Department of Mathematics and Statistics, Utah State University, 3900 Old Main Hill, Logan, Utah 84322

E-mail address: anle@cc.usu.edu 